\title{
EL PUENTE DE PIEDRA DE GRECIA: ¿UN ARCO O UN PUENTE NATURAL?
}

\author{
Rolando Mora \\ Escuela Centroamericana de Geología, Universidad de Costa Rica \\ E-mail: rmorach@geologia.ucr.ac.cr
}

(Recibido 8/11/04; Aceptado 27/1/05)

\begin{abstract}
The natural and selective erosion of the volcanic rocks that compound it has formed Puente de Piedra (Rock Bridge). This is a nice example on the manifestation of the erosion processes in Nature. This natural structure might be considered as a natural arch or as a natural bridge. Its base shows evidences of strength increment due to compression. Taxonomically, Puente de Piedra is a Meandering Natural Bridge if all its attributes are considered.

Keywords: Rock bridge, Costa Rica, natural arch, meandering natural bridge.

RESUMEN: El Puente de Piedra se ha formado por la remoción natural y selectiva de las rocas volcánicas que lo forman, es decir, es el resultado de la manifestación de procesos erosivos. Esta estructura natural puede considerarse como un arco natural o como un puente natural. Su base presenta el efecto de incremento de resistencia por compresión. El Puente de Piedra se ha clasificado, taxonómicamente, como un Puente Natural Meándrico de acuerdo con sus atributos.

Palabras clave: Puente de piedra, Costa Rica, arco natural, puente natural meándrico.
\end{abstract}

\section{INTRODUCCIÓN}

El Puente de Piedra es una estructura natural con un fuerte sabor a leyenda, "el puente que construyó el Diablo", según la cultura popular del Cantón de Grecia y que llena de tanto orgullo a sus habitantes. Este puente se ubica en la comunidad de Puente de Piedra de Grecia, sobre el río Poró, en las coordenadas Lambert Costa Rica Norte 500500 E y 224995 N, según la hoja topográfica Puente de Piedra, escala 1:10000 del I.G.N. (Fig.1).

\section{ANÁLISIS}

\section{¿Es el Puente de Piedra un arco natural?}

Un arco natural es un afloramiento de roca que tiene un agujero que lo atraviesa completamente y que es formado por la remoción natural y selectiva de la roca, la cual deja un marco relativamente intacto (Wilbur, 2003).

El Puente de Piedra de Grecia está compuesto por rocas volcánicas piroclásticas de flujo y de caída (Mora et al., 2003). La base del puente está parcialmente cubierta por el agua del río Poró (Figs. 2 y 3 ). 


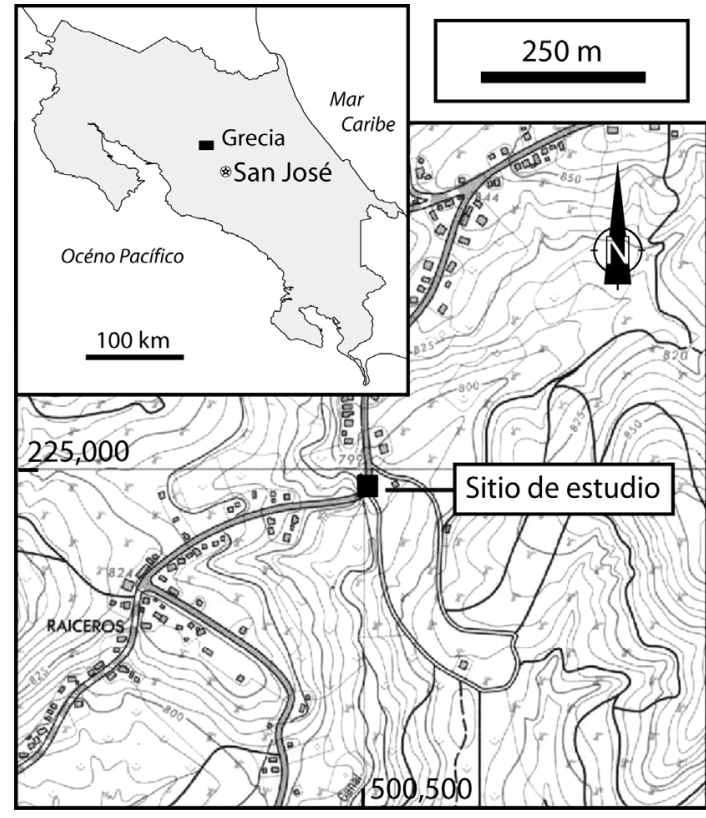

Fig. 1: Ubicación geográfica del Puente de Piedra, Grecia.

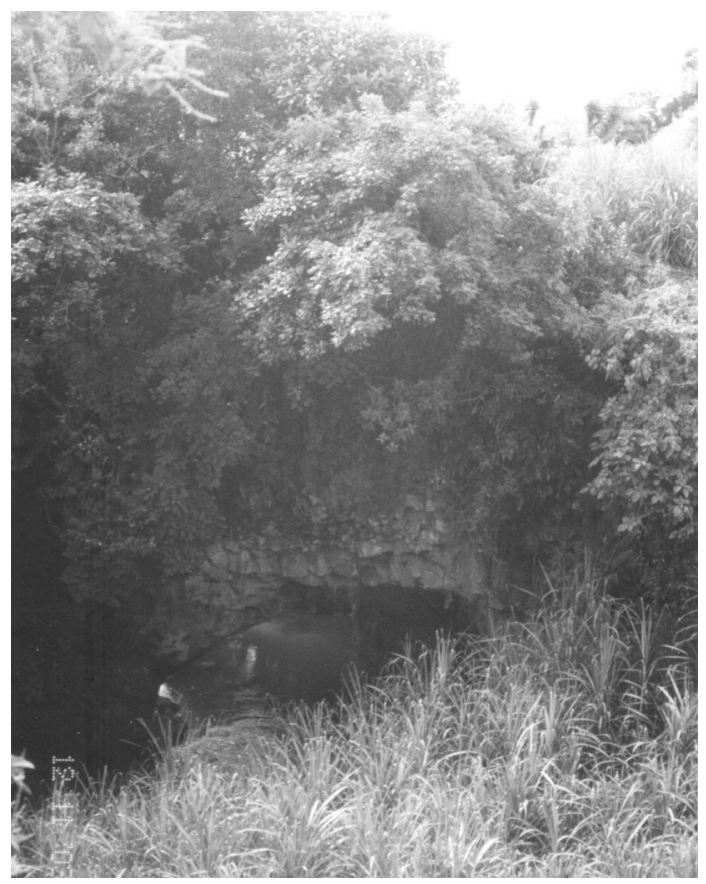

Fig. 3: Vista panorámica del Puente de Piedra desde el Oeste.

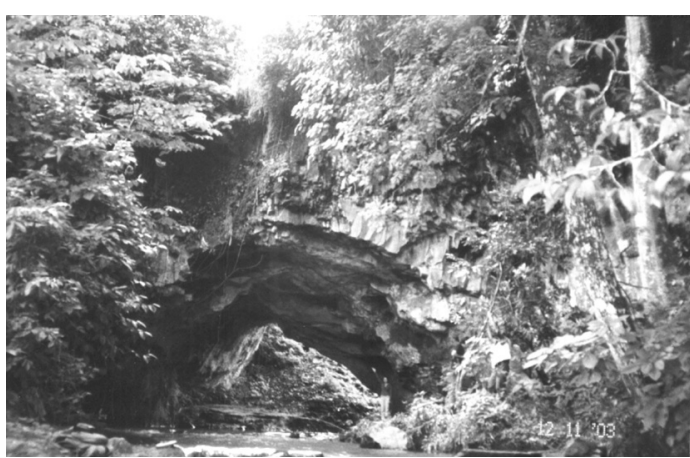

Fig. 2: Vista panorámica del Puente de Piedra desde el Este.

El agujero a través de la roca cumple con la definición matemática o topológica de un agujero: una superficie con un agujero tiene un genero de 1 , es decir, es posible dibujar en la superficie una curva cerrada, simple, sin intersecciones, sin separar la superficie en regiones diferentes. Un arco natural con un único agujero es topológicamente equivalente con un toroide (Wilbur, 2003).

El agujero del Puente de Piedra se ha formado por la remoción natural y selectiva de las rocas que lo componen, como resultado de procesos erosivos. El marco de roca que rodea al agujero se presenta relativamente intacto, su sección superior es un macizo rocoso intensamente fracturado y la inferior una roca volcánica extremadamente blanda (Mora et al., 2003).

Todo lo anteriormente expuesto conduce a concluir que, en efecto, el Puente de Piedra puede considerarse como un arco natural.

\section{¿Es el Puente de Piedra un puente natural?}

Un puente natural es un tipo de arco natural. En general, un puente natural se distingue de otros tipos de arcos naturales cuando cuenta con uno o más de los siguientes atributos (Wilbur, 2003):

- una corriente de agua, como un río, ha sido claramente el agente principal en la formación del agujero (atributo genético), una corriente de agua, como un río, fluye a través del agujero (atributo contextual), 
- $\quad$ es utilizado o ha sido utilizado por las personas como un puente (atributo antropomórfico),

- tiene la apariencia general de un puente construido por los seres humanos, una sección superior plana sobre una abertura arqueada (atributo antropomórfico).

Cada uno de los atributos arriba indicados se pueden aplicar al caso del Puente de Piedra, por lo cual este arco natural se puede clasificar como un puente natural.

Wilbur (2003) recomienda el uso de la siguiente definición en cualquier descripción o clasificación de arcos naturales: un puente natural es un tipo de arco natural donde una corriente de agua, como un río, claramente ha sido el agente principal en la formación del agujero.

\section{Antiguiedad del Puente de Piedra}

Los arcos naturales se desarrollan en rocas que son mucho más antiguas que la estructura misma. El Puente de Piedra está compuesto por material derivado de explosiones volcánicas (Mora et al., 2003) que ocurrieron hace 320000 a 390000 años (Marshall et al., 2003).

Es claro que esta estructura es de vida corta en la escala del tiempo geológico y que se trata de un proceso muy reciente, pero actualmente no hay manera de determinar exactamente cuando se formo el Puente de Piedra. Según Wilbur (2003), se puede decir que ningún arco natural es mayor de aproximadamente 30000 años, la mayoría tiene probablemente entre 5000 y 15000 años de edad.

\section{Formación del Puente de Piedra}

Un arco natural se forma solamente bajo circunstancias muy especiales, entre las que se incluyen el tipo o tipos de roca presentes, la forma del afloramiento y la combinación de procesos erosivos que actúan sobre él (Wilbur, 2003).

El Puente de Piedra se ha formado por la remoción natural y selectiva de las rocas, como se establece en la definición de un arco natural. Los procesos naturales que han conducido a su formación son exclusivamente procesos de erosión, los cuales pueden remover la roca macroscópica y microscópicamente y de manera muy efectiva.

Si consideramos el depósito ignimbrítico, que compone la parte superior del puente, como una forma del terreno relativamente plana (tabular), probablemente el río Poró desarrolló un proceso de serpenteo alrededor de las irregularidades del mismo depósito (Fig. 4 a). Esto condujo al desarrollo de curvaturas del cauce, las que tienden a cerrarse sobre si mismas y que se conocen como meandros. Posteriormente, a raíz de un levantamiento tectónico regional, el río inicia un proceso de incisión vertical para mantenerse a una elevación constante, mientras el levantamiento se manifiesta; de esta manera se desarrollan los meandros encajados (Fig. 4 b). La sección donde el meandro prácticamente se cierra se conoce como el cuello y para los meandros encajados el cuello es una pared de roca alta y delgada. Es en este punto donde otros procesos erosivos crean una abertura a través de la pared para así iniciar la formación del arco natural.
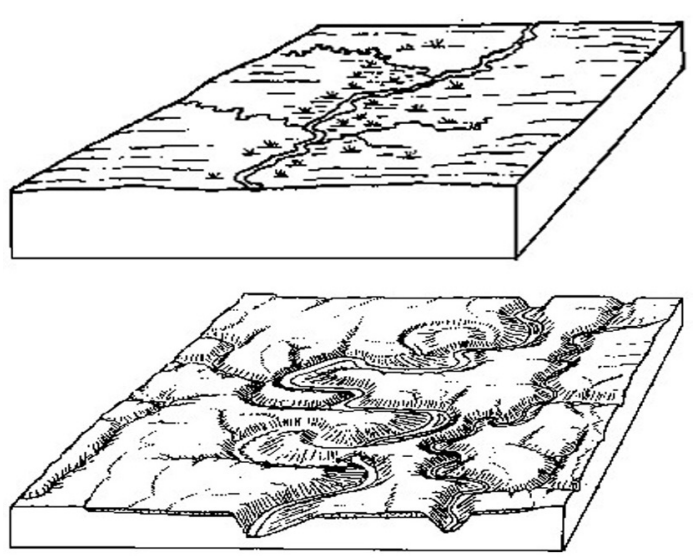

Fig. 4: a) Inicio del proceso de serpenteo alrededor de las irregularidades del un depósito tabular. b) Desarrollo de meandros encajados y formación de un arco natural (Strahler \& Strahler, 1992)

El agua río Poró inició el proceso de erosión microscópica de la toba que compone la base del puente, disolviendo el cementante existente 
entre los granos del material, el cual tiene su origen en la depositación a temperaturas elevadas de flujos piroclásticos (Mora et al., 2003). De esta manera se inicia la destrucción de la matriz y el arrastre de los granos de la toba, lo que da paso a un proceso de tubificación o erosión interna del material como lo mencionan Mora et al. (2003).

Al avanzar la erosión interna, la parte superior del afloramiento, un macizo rocoso ignimbrítico intensamente fracturado (Mora et al., 2003), queda expuesta a un proceso de erosión macroscópica, en donde las piezas de roca pueden moverse, unas con respecto a las otras, bajo la acción de la fuerza de gravedad o la presión del agua. Este proceso de erosión macroscópica es el que ha dominado para dar la forma actual al Puente de Piedra.

\section{Componentes del Puente de Piedra}

La abertura (agujero) del Puente de Piedra es el volumen (de aire) que llena el hueco del puente. Las entradas son las superficies que limitan la abertura del resto de la atmósfera, en la figura 5 se aprecia la entrada Este del Puente de Piedra. El dintel es el volumen de roca sin soporte. La base es la superficie bajo la abertura. El estribo es el volumen del marco de roca que soporta el dintel pero no es parte de la base.

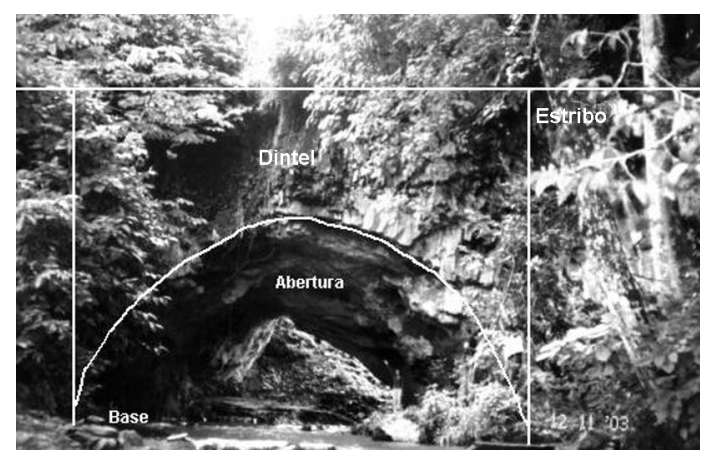

Fig. 5: Componentes del Puente de Piedra.

\section{Incremento de resistencia por compresión.}

El peso del macizo rocoso fracturado actúa comprimiendo la toba masiva de la base del puente; esta fuerza se desarrolla en dirección vertical. Cuando la roca es soportada en un arco, las líneas de fuerza se desvían de la vertical (Wilbur, 2003), curvándose alrededor del arco y retomando su dirección vertical bajo el mismo (Fig. 6). Este proceso causa un incremento de la resistencia del material que está siendo comprimido (Wilbur, 2003), en nuestro caso la toba masiva.

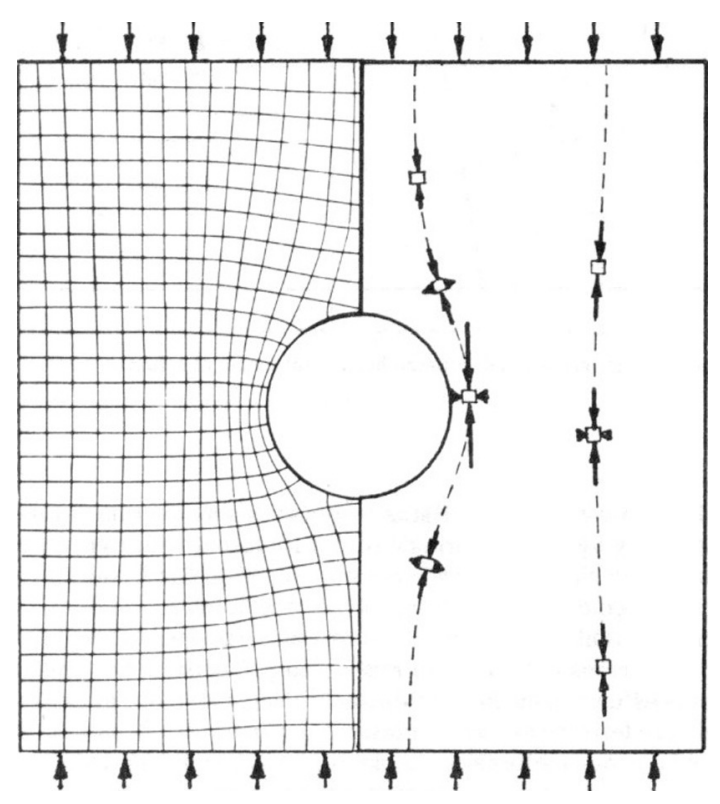

Fig. 6: Trayectoria de los esfuerzos principales mayor y menor, alrededor de un orificio circular (Hoek \& Brown, 1985).

En la toba masiva la compresión causa la deformación de los granos en la matriz del depósito, lo cual se suma al efecto cementante e incrementa su fuerza de adhesión, es decir, la roca se torna más dura y más resistente a la erosión (Wilbur, 2003). Este proceso se refleja en los ensayos de resistencia al corte realizados por Mora et al. (2003), pero es atribuido únicamente al efecto de depositación en caliente del flujo piroclástico por los mismos autores. El proceso de incremento de resistencia por compresión 
desarrolla un dintel más resistente a la erosión y, por lo tanto, alarga la vida útil del arco natural (Wilbur, 2003).

\section{Clasificación y taxonomía del Puente de Piedra}

Antes de presentar la taxonomía recomendada para el Puente de Piedra, se presentan algunos atributos que son útiles en la descripción de este arco natural.

El Puente de Piedra se ha desarrollado por la acción de una corriente fluvial y puede considerarse como inundado, pues el agua del río cubre parte del marco de roca bajo el agujero. Estos son atributos contextuales de la estructura.

La abertura es semicircular, donde las entradas son paralelas, horizontales y separadas por una distancia que es menor que la luz del arco. Se puede considerar como un arco plano, pues la parte superior del dintel es horizontal y plana; así mismo, el dintel es masivo ya que es de mayor tamaño que el agujero. Los anteriores son atributos morfológicos de la estructura.

Las dimensiones de la estructura incluyen 14 $\mathrm{m}$ de ancho y $7 \mathrm{~m}$ de alto del agujero, así como, 12 $m$ de separación entre las entradas. Se trata de un arco de tamaño significativo, pues el producto de las dimensiones ortogonales del agujero es mayor de $10 \mathrm{~m}^{2}$. Estos son atributos métricos del arco.

Los atributos geológicos indican que el arco se ha desarrollado en tobas e ignimbritas, las cuales pertenecen a la Unidad Ignimbrita Puente de Piedra y a las Subunidades Toba Masiva Puente de Piedra e Ignimbrita Columnar Puente de Piedra respectivamente (Mora et al., 2003).

Dentro de los atributos genéticos se puede decir que el arco se desarrolló a partir de un meandro encajado, con la contribución de procesos de erosión diferencial por infiltración y/o flujo de agua, colapso del techo y actualmente el incremento de resistencia por compresión de la base.

Desde el punto de vista de sus atributos de madurez, el Puente de Piedra se puede considerar como un arco adulto, pues hay suficiente evidencia sobre su modo de formación, así como, de su desarrollo posterior.
A continuación se presenta la taxonomía de los arcos naturales recomendada por Wilbur (2003):

- Arco Natural Abandonado

-Arco Natural en Arco

- Arco Natural Cumbre de Roca

- Caverna

- Arco Natural en Lava

- Arco Natural Columnar

- Arco Natural Apoyado

- Arco Natural Protegido

- Arco Natural en Alcoba

- Arco Natural en Contrafuerte

- Arco Natural en Cueva

-Arco Natural en Aleta

- Puente Natural Meándrico

- Arco Natural en Marmita

- Arco Natural Marino

- Arco Natural en Cascada

- Arco Natural Irregular

El Puente de Piedra se ha clasificado como un Puente Natural Meándrico, ya que el mismo se asocia con una corriente fluvial activa, el agujero es semicircular y el dintel arqueado. La característica plana de la parte superior del dintel y arqueado en su parte inferior, recuerda un puente construido por los seres humanos.

El agua del río Poró rellena parcialmente el agujero, pero no se debe considerar parte del mismo, se considera parte de la base. El Puente de Piedra presenta un dintel horizontal, soportado por dos estribos.

\section{Identificación del Puente de Piedra}

El estándar propuesto para etiquetar los arcos naturales está basado en sus localizaciones en el sistema UTM (Universal Transverse Mercator) y el WGS84 (World Geodetic System of 1984) (Wilbur, 2003). La etiqueta de referencia completa para un arco natural incluye sus coordenadas UTM con el prefijo "NABSQNO", es decir, la etiqueta tiene el formato NABSQNO Z-E-N, donde Z es la zona UTM, E el este UTM y N el norte UTM (Wilbur, 2003). 
La etiqueta de referencia del Puente de Piedra es la siguiente:

NABSQNO 16-0793041-1111197

Costa Rica, Provincia de Alajuela, Cantón de Grecia, Distrito Puente de Piedra.

\section{CONCLUSIONES}

El Puente de Piedra es un arco natural, el cual clasifica como un puente natural. Actualmente no hay manera de determinar exactamente cuando se formó el Puente de Piedra.

El Puente de Piedra se ha formado por la remoción natural y selectiva de las rocas que lo componen. Los procesos naturales que han conducido a su formación son exclusivamente procesos de erosión, los cuales pueden remover la roca macroscópica y microscópicamente y de manera muy efectiva.

El Puente de Piedra se ha clasificado como un Puente Natural Meándrico, ya que el mismo se asocia con una corriente fluvial activa, el agujero es semicircular y el dintel arqueado. La característica plana de la parte superior del dintel y arqueado en su parte inferior, recuerda un puente construido por los seres humanos.

\section{REFERENCIAS}

HOEK, E. \& BROWN, E.T., 1985: Excavaciones subterráneas en roca. - 634 págs. McGraw-Hill, México.

MARSHALL, J., IDLEMAN, B., GARDNER, T. \& FISHER, D., 2003: Landscape evolution within a retreating volcanic arc, Costa Rica, Central America. - Geology, 31(5): 419-422.

MORA, R., CHAVES, J., ALVARADO, M., CAMACHO, D., MURILLO, D., BARRANTES, M. \& HERRERA, P., 2003: Caracterización geológica y geotécnica del Puente de Piedra, Grecia, Alajuela: de la leyenda a la realidad geológica. 15 págs. G-4213 Mecánica de Rocas, Escuela Centroamericana de Geología, Universidad de Costa Rica [Inf. inédito].

STRAHLER, A.H. \& STRAHLER, A.N., 1992: Modern physical geography. - 4a ed. 638 págs. John Wiley \& Sons, New York.

WILBUR, J.H., 2003: Natural arch information. http://www.naturalarch.info/ . Página consultada el 03-05-2004. 